\title{
Durability of Two R-Phase Rotary Systems used in the Mechanical Instrumentation of Dilacerated Root Canals
}

\author{
Flavia D Vivacqua ${ }^{1}$, Marcella Canto ${ }^{1}$, George T Candeiro $^{2}$, Bruno $\mathrm{C}$ de Vasconcelos ${ }^{3}$, Nilton Vivacqua-Gomes ${ }^{4}$
}

\begin{abstract}
Aim: This study was aimed to analyze, through an ex vivo test, the durability of two rotary systems used in curved root canals of extracted molars. Materials and methods: At first, 150 upper and lower molars were selected, presenting curvature at least in one of the root canals. They underwent access cavity and radiographed with a \# 15 file into the canal to determine their maximum curvatures, classified as schneider. The teeth with one or more curved canals were included in this study. Finally, 120 curved root canals were statistically divided into six sets of 20 curved canals. Two rotatory systems, K3 XF (RCS sequence) and twisted file (Large Kit), both treatment R-Phase, were analyzed. Three kits were used for each system, each one instrumented one of the six sets of 20 curved canals. File deformations were noted, and the files were used until a fracture occurs or until completion of 20 canals preparation. Fractured files before the end of the instrumentation of the 20 canals were replaced, continuing the full instrumentation set. Statistical analysis was performed by analysis of variance (ANOVA) $(p>0.05)$, and a qualitative assessment was also made. Results: It was found that there was no statistical difference between the K3 XF and TF systems regarding fracture.

Conclusion: There were no statistical differences between the K3 XF systems in the RCS sequence and the twisted file kit Large system, as to the number of instrumented conduits to the deformation or fracture.

Clinical significance: The findings of this study reinforce that, it is necessary to choose files that support curved roots preventing accidents inside the root canal. Therefore, it is important for professionals to have knowledge and command of these protocols to obtain more satisfactory results.
\end{abstract}

Keywords: Endodontics, Root canal preparation, Rotary endodontics.

The Journal of Contemporary Dental Practice (2019): 10.5005/jp-journals-10024-2530

\section{INTRODUCTION}

$\mathrm{O}$ ne of the main objectives of endodontic file manufacturers is to produce instruments with increased resistance to separation, including the nickel-titanium (NiTi) files used in the mechanical instrumentation of root canals. Thus, with new methods of producing the instruments and with the resistance improved, the use of the instruments would be safer. ${ }^{1}$

It is known that the area of the instrument prone to fatigue coincides with marks and depressions left in the file by the machining process. ${ }^{2}$ Moreover, an important point to be taken into account when talking about instrument fractures is the size of the root canal. However, in this regard, there are only a few studies correlating dilacerated root canals and instrument fracture. ${ }^{3}$

The SybroEndo manufacture process, available since 2008, is related to the production of the twisted file system, which presents a triangular transversal section and high cutting efficiency. The twisted file system is the only one with NiTi instruments produced by torsion, a process allowed by a thermic treatment named $R$ phase. Such treatment is applied to the instruments after the conclusion of the machining process with the aim of transforming molecular phase of the metal in a structure different from the original. ${ }^{4}$

These improvements in technology and manufacture processes culminated in a new generation of endodontic files. The already existent K3 (SybronEndo, Orange, CA, EUA) were improved to K3 XF that presented higher flexibility and resistance to fatigue. They are now produced by conventional machining with the triple-helical transversal section, maintaining the original design. However, in the new version, the $\mathrm{R}$ phase treatment corrects the fragilities of machining process of the old version of the instrument. ${ }^{4}$

The availability of new instruments that supposedly present improved characteristics for clinical use is increasing. However, there are few studies reproducing their use and checking their
${ }^{1,4}$ School of Dentistry of Ceará, São Leopoldo Mandic University, Postgraduation Program in Endodontics, Campus Fortaleza, Fortaleza, CE, Brazil

${ }^{2}$ Universidade Christus, Department of Dentistry, Postgraduation Program in Dental Sciences, Fortaleza, CE, Brazil

${ }^{3}$ School of Dentistry of Sobral, Federal University of Ceará, Campus Sobral, Sobral, CE, Brazil

Corresponding Author: Flavia D Vivacqua, School of Dentistry of Ceará, São Leopoldo Mandic University, Postgraduation Program in Endodontics, Campus Fortaleza, Fortaleza, CE, Brazil, Phone: +55 85 982161645, e-mail: fladarius@gmail.com

How to cite this article: Vivacqua FD, Canto M, Candeiro GT, de Vasconcelos BC, Vivacqua-Gomes N. Durability of Two R-Phase Rotary Systems used in the Mechanical Instrumentation of Dilacerated Root Canals. J Contemp Dent Pract 2019;20(4):405-408.

Source of support: Nil

Conflict of interest: None

resistance, mainly in dilacerated canals. Therefore, the aim of this investigation is to evaluate the durability of the rotary systems $\mathrm{K} 3$ $\mathrm{XF}$ and TF, both with the $\mathrm{R}$ phase thermic treatment.

\section{Materials and methods}

After ethical approval by the research ethics committee, human molars extracted for therapeutic reasons were selected. As previous studies had already shown that the instruments to be used in this investigation present only slight variations in regard to fracture and cyclical fatigue, three kits were mounted.

\section{Sample Selection}

Molars presenting one or more root canal with a dilaceration between $30^{\circ}$ and $90^{\circ}$ were selected by the Schneider method 
and circumference radius (2-9 $\mathrm{mm})$. A sample of 120 roots dilacerated canals were selected for this study. Each instrument kit was used to prepare 20 root canals. After being disinfected with sodium hypochlorite $2.5 \%$ for 30 minutes, the teeth were kept in physiological solution for one week.

\section{Preparation of the Specimen}

The root canals were numbered and randomly assigned in six groups and allocated in one kit of one of the two instrument systems. The root canals were statistically evaluated in terms of their radius and curvature angle, and no differences were found (ANOVA). In the group twisted file (TF), the instruments of the system Twisted File Large (SybronEndo, Orange, CA, EUA) were used. In the group, K3 XF instruments were used in the RCS sequence after the access cavity, the teeth were prepared under irrigation with $2 \%$ chlorhexidine gluconate in the gel (CHX; BioPhormula, Fortaleza, CE, Brazil) followed by irrigation with $0.9 \%$ physiological solution. The opening of the root canals was prepared with CP Drill (Helse Ultrasonics, Santo Rosa do Viterbo, $\mathrm{SP}$, Brasil). The working length was established at the $1 \mathrm{~mm}$ before the apex foramen.

All the procedures were performed by a single trained operator. The motor elements (SybronEndo) were used to trigger the instruments. Standardized speed and torque were established as follows:

\section{Group TF-KIT Large}

Cervical and medium thirds of the root canals were prepared with the instrument $25 / .10$ (350 RPM e $2 \mathrm{~N}$ ). In the beginning of the apical third, the instrument 25/.08 (350 RPM e $2 \mathrm{~N}$ ) was used, while in the working length the instrument 25/.06 (300 RPM e $1 \mathrm{~N}$ ) was employed.

\section{Group K3 XF-RCS}

The cervical and medium thirds of the root canals were prepared with the instrument 25/.08 (350 RPM e $2 \mathrm{~N}$ ). In the sequence, the instruments $15,20,25,30 / .04$ (300 RPM e 1,5 N) e 20/.06 (300 RPM e 1,5 N) were employed in the working length.

After each instrument change, irrigation was performed with 5 $\mathrm{mL}$ of physiologic solution. When the endodontic file was not able to reach the working length, the instrument spirals were cleaned, and irrigation was performed again. The endodontic file was inserted into the canal as much as it was needed to reach the determined working length.

The endodontic files were qualitatively evaluated after each use in regard to their deformation and separation by inspection with a magnifying glass (LED Headlight Titan) under a $3.5 \times$ magnification. When deformation or separation was observed, the place of such alteration and the number of times that the instrument had been used were registered. When an instrument fractured, it was replaced by new ones to finish the instrumentation.

Table 1: Means, standard deviation, minimum and maximum number of root canals instrumented per system

\begin{tabular}{lllll}
\multicolumn{5}{c}{ number of root canals instrumented per system } \\
\hline & $\begin{array}{l}\text { Until deformation } \\
\text { mean }(S D)\end{array}$ & $\begin{array}{l}\text { Min- } \\
\text { max }\end{array}$ & $\begin{array}{l}\text { Until fracture } \\
\text { mean }(S D)\end{array}$ & $\begin{array}{l}\text { Min- } \\
\text { max }\end{array}$ \\
\hline TF & $11.00 \mathrm{a}( \pm 5.72)$ & $3-20$ & $17.89^{\mathrm{a}}( \pm 3.76)$ & $10-20$ \\
K3 XF & $15.35 \mathrm{a}( \pm 6.12)$ & $2-20$ & $16.58^{\mathrm{a}}( \pm 4.46)$ & $6-20$ \\
\hline
\end{tabular}

\section{Statistical Analysis}

Normality was checked, and the data were analyzed by ANOVA, considering two outcomes, instrument deformation, and dilaceration.

\section{Results}

The mean of root dilaceration was $49.6^{\circ}$ with a radius of $6,3 \mathrm{~mm}$ in both experimental groups. The comparison between groups in regard to deformation and fracture is shown in Table 1. No statistically significant differences were found in both analyses when comparing the groups $(p>0.05)$. Only two instruments of the TF group fractured during the instrumentation. Any endodontic file fractured before being used less than 10 times. The file $25 / .10$ exhibited resistance until its 20 th use. All the files $25 / .08$ only fractured after the 10th use, resisting fatigue without showing any sign of prior deformation. Regarding the file 25/.06, deformation was seen with the instrumentation of 4-11 root canals, however, any of these instruments fractured (Table 2).

Regarding the K3 XF system, instruments enabled the instrumentation of six curved root canals with no fracture of the instrument. The file 25/.08 was fractured two times, one fracture occurred after the sixth time of use (kit 3 ) and the other after being used 14 times (kit 2). In both, fractures affected the intermediate portion near the tape during the instrumentation of the cervical and middle thirds of the root canal. Two files 15/.04 remained intact after instrumentation, and one of them (kit 2) deformed after being used 11 times until the final fracture when preparing the 13th root canal. Instruments 20/.04 and 20/.06 were more resistant until the end of the experimental protocol. Conversely, instrument 25/.04 was the least durable as just one 25/.04 file (kit 3) remained intact until the end of the instrumentation. Two 30/.04 files did not fracture after the experimental protocol whilst only one deformed after the third use (Table 3).

\section{Discussion}

The analysis of longevity until a fracture occurrence is justified due to the reuse of endodontic files in an everyday clinical condition, thus determining a safe number of uses is relevant. Wolcott et al. ${ }^{5}$ considered that a fracture of endodontic instruments is an inherent risk of the endodontic treatment and the number of uses is a determining factor of this type of accident.

According to Bhagabati et al., ${ }^{6}$ the influencing factors for endodontic instrument fractures were frequently described in the literature individually, however, they act simultaneously during the root canal instrumentation, which supports the use of extracted human teeth for experimental protocols. ${ }^{7-9}$

The anatomy of radicular canals, mainly diameter, curvature radius and angle, number of instruments used, transversal section, torque, rotation speed, experience, preparation

Table 2: Durability (number of root canals) of three kits of TF files during instrumentation of 20 root canals

\begin{tabular}{llll}
\hline TF & $25 / 0.10$ & $25 / 0.08$ & $25 / 0.06$ \\
\hline Kit 1 & 20 & $20^{*}$ & $20^{*}$ \\
Kit 2 & 13 & 7 & 10 \\
& 9 & $18^{*}$ & \\
Kit 3 & 20 & $20^{*}$ & $20^{*}$ \\
\hline
\end{tabular}

*Deformed during use. Values 7 and 9 refer to files that replaced fractured ones to enable finishing the instrumentation of 20 root canals 
Durability of Two R-Phase Rotary Systems used in the Mechanical Instrumentation of Dilacerated Root Canals

Table 3: Durability (number of root canals) of 3 kits of K3XF files during instrumentation of 20 root canals

\begin{tabular}{lllllll}
\hline K3 XF & $25 / .08$ & $15 / .04$ & $20 / .04$ & $25 / .04$ & $30 / .04$ & 19 \\
\hline Kit 1 & 20 & 20 & 20 & 7 & 13 & 19 \\
Kit 2 & 14 & $13^{*}$ & 19 & 19 & 19 & 19 \\
Kit 3 & 6 & $7^{*}$ & 19 & 19 & $11^{*}$ & 18 \\
& 6 & 19 & & & 8 & \\
\hline
\end{tabular}

*Deformed during use. Values 7 and 9 refer to files that replaced fractured ones to enable finishing the instrumentation of 20 root canals

technique, manufacturing process, instrument design, and surface modification of the metallic alloy are some factors reported in the literature that affect endodontic instruments and could contribute to the fractures. ${ }^{4,6,10-13}$

Thermal processing of the systems used, namely R-Phase, seems to play a role in eliminating the drawbacks of the machining process and yielding instruments with a superior mechanical resistance. ${ }^{1,14-16}$ According to Kim et al., ${ }^{17}$ thermally treated NiTi files, M-Wire, R-Phase, or CM-Wire instruments demonstrated improved resistance to cyclic fatigue in comparison with conventional $\mathrm{NiTi}$ files, showing the mechanical superior features of these modified alloys.

Another advantage regarding the heating process of R-Phase was described by Zhao et al. ${ }^{18}$ who evaluated through microcomputed tomography (microCT) the preparation of the radicular canal by Hyflex CM, TF, and $\mathrm{K} 3$ files. The TF system removed higher amounts of dentin $(p<0.05)$, while no significant difference between Hyflex CM and K3 was found. The TF system also significantly reduced over instrumentation compared with the $\mathrm{K} 3$ system but with no difference in relation to the Hyflex CM system.

Here, the TF system files enabled the instrumentation of 10 radicular canals without a single fracture, compared with the six canals prepared by using the K3 XF system, no statistical difference was observed between groups. Similar findings were described by Kim et al. ${ }^{17}$ and Rodrigues et al. ${ }^{19}$ when compared the resistance to fatigue of machined NiTi rotatory instruments with TF files. The latter showed higher resistance values to cyclic fatigue. Likewise, Bhagabati et al. ${ }^{6}$ evaluated the resistance to fracture in vitro of three machined systems (ProFile, Mtwo, K3) and TF. The latter presented increased resistance and the authors associated its better performance to the thermal treatment it received as well as to its electropolishing. However, PérezHigueras et al. ${ }^{13}$ compared the resistance to cyclic fatigue with the continuous and reciprocating kinematics of $\mathrm{K} 3, \mathrm{~K} 3 \mathrm{XF}$, and TF systems. K3 XF system obtained the best performance, followed by TF systems. This can be due to the transversal section shape of these instruments, which aids in dissipating the strength of the cutting edges of the instruments.

Caballero et al. ${ }^{9}$ tested TF instruments (25/0.08) comparing them with Reciproc (R25) regarding the instrumentation of root canals with curvature angles between $15^{\circ}$ and $30^{\circ}$ as well as radii ranging from 4 to $5 \mathrm{~mm}$. The authors observed that instruments presented defects such as plastic deformation and changes in the cutting edge after using TF files six times, and these changes increased even more after using the instruments 12 times. This corroborates with our results which showed that TF instruments were used 11 times until deformation.

It is important to highlight that extrapolating results obtained in preclinical protocols to a clinical scenario should be done carefully as many variables can be controlled, mainly related to the position of the tooth in the dental arch of the patient, opening of the mouth and tiredness during the endodontic treatment. However, this study was carried out with extracted human teeth to approximate our conditions to the clinical ones at the most. The results obtained point to the possibility of reuse of the instruments, however, in curved channels, should be considered, instruments with heat treatment, small tip, and small conicity, should be preferably new or little used. Nonetheless, our results do not recommend reusing files after deformations occur.

\section{Conclusion}

Under the conditions of this study, there were no statistically significant differences between K3 XF and twisted file kit systems regarding the rotatory sequence for safe curvatures and the number of radicular canals prepared until deformation or fracture. The TF system instrumented ten root canals while K3XF system prepared six canals until fracturing.

\section{References}

1. Gambarini G, Plotino G, Grande NM, et al. Mechanical properties of nickel-titanium rotary instruments produced with a new manufacturing technique. Int. Endod J 2011;44(4):337-341.

2. Castello-Escriv. R, Alegre-Domingo T, Faus-Matoses V, et al. In vitro comparision of cyclic fadigue resistence of protaper, waveone and twisted file. J Endod 2012;38(11):1521-1524.

3. Cheung GSP, Oh SH, Há JH, et al. Effect of torsional loading of nickel-titanium instruments on cyclic fatigue resistance. J Endod 2013;39(12):1593-1597.

4. Gambarini G, Gergi R, Naaman A, et al. Cyclic fatigue analysis of twisted file rotary $\mathrm{NiTi}$ instruments used in reciprocating motion. Int Endod J 2012;45(9):802-806.

5. Wolcott S, Wolcott J, Ishley D, et al. Separation incidence of protaper rotary instruments: A large cohort clinical evaluation. J Endod 2006;32(12):1139-1141.

6. Bhagabati N, Yadav S, Talwar S. An in vitro cyclic fatigue analysis of different endodontic nickel-titanium rotary instruments. J Endod 2012;38(4):515-518.

7. Vieira MVB. A influência da geometria de canais artificiais na fratura de instrumentos de NiTi por flexão rotativa contínua: [Tese]. Rio de Janeiro: Faculdade de Odontologia da Universidade Estácio de Sá; 2012. Portuguese.

8. Capar ID, Ertas H, Ok E, et al. Comparative study of different novel nickel-titanium rotary systems for root canal preparation in severely curved root canals. J Endod 2014;40(6):852-856.

9. Caballero H, Rivera F, Salas H. Scanning electron microscopy of superficial defects in twisted files and reciproc nickel-titanium files after use in extracted molars. Int Endod J 2015;48(3):229-235.

10. Yared G. Canal preparation using only one $\mathrm{Ni}-\mathrm{Ti}$ rotary instrument: preliminary observations. Int Endod J 2008;41(4):339-344.

11. Wu J, Lei G, Yan M, et al. Instrument separation analysis of multi-used ProTaper Universal rotary system during root canal therapy. J Endod 2011;37(6):758-763. 
12. Wan J, Rasimick BJ, Musikant BL, et al. A comparison of cyclic fatigue resistance in reciprocating and rotary nickel-titanium instruments. Aust Endod J 2011;37(3):122-127.

13. Pérez-Higueras JJ, Arias A, Macorra J, et al. Differences in cyclic fatigue resistance between ProTaper Next and ProTaper Universal instruments at different level. J Endod 2014;40(9):1477-1481.

14. Ebihara A, Yahata Y, Miyara K, et al. Heat treatment of nickel-titanium rotary endodontic instrument effects on bending properties and shaping abilities. Int Endod J 2011;44(9):843-849.

15. Lopes HP, Vieira MVB, Elias CN, et al. Influence of the Geometry of curved artificial canals on the fracture of rotary nickel-titanium instruments subjected to cyclic fatigue tests. J Endod 2013;39(5):704707.
16. Plotino G, Costanzo A, Grande NM, et al. Experimental evaluation on the influence of autoclave sterilization on the cyclic fatigue of new nickel-titanium rotary instruments. J Endod 2012;38(2):222-225.

17. Kim HC, Yum J, Hur B, et al. Cyclic fatigue and fracture characteristics of ground and twisted nickel-titanium rotary files. J Endod 2010;36(1):147-152.

18. Zhao $D$, Shen $Y$, Peng B, et al. Micro-computed tomography evaluation of the preparation of mesiobuccal root canals in maxillary first molars with hyflex cm, twisted files, and k3 instruments. J Endod 2013;39(3): 385-388.

19. Rodrigues RCV, Lopes HP, Elias CN, et al. Influence of different manufacturing methods on the cyclic fatigue of rotary nickeltitanium endodontic instruments. J Endod 2011;37(11):1553-1557. 